\title{
Spectroscopy of Rashba spin splitting in InSb quantum wells
}

\author{
G. A. Khodaparast,* R. E. Doezema, S. J. Chung, K. J. Goldammer, and M. B. Santos \\ Department of Physics and Astronomy and Center for Semiconductor Physics in Nanostructures, University of Oklahoma, \\ Norman, Oklahoma 73019, USA
}

(Received 2 July 2004; published 22 October 2004)

\begin{abstract}
We use electron spin resonance in the far infrared to probe the Landau-level spin splitting in symmetric and asymmetric InSb quantum wells. The asymmetric wells exhibit a strong deviation in behavior from the symmetric wells at low magnetic fields with apparent $g$ factors far in excess of the bulk $g$ factor of InSb. These asymmetry-induced shifts in the spin resonance depend on Landau-level index as predicted by the BychkovRashba model. We analyze the shifts to extract values for the Rashba parameter.
\end{abstract}

DOI: 10.1103/PhysRevB.70.155322

PACS number(s): 78.67.De, 78.20.Ls, 71.70.Ej

The study of electron spin phenomena in semiconductor heterostructures has intensified dramatically in recent years. The field, "spintronics" has emerged with the purpose of developing devices combining the charge and spin degrees of freedom. In particular, spin splitting caused by bulk inversion asymmetry and structural inversion asymmetry (SIAoften called Rashba splitting) has attracted much attention. Understanding the spin-orbit interaction caused by SIA, which is predicted to lead to spin splitting even without an applied magnetic field, ${ }^{1,2}$ is important for developing spinbased devices. To date, experimental evidence for Rashba splitting in two-dimensional electron systems (2DESs) has been chiefly confined to the observed beating of Shubnikov-de Haas $(\mathrm{SdH})$ oscillations ${ }^{3,4}$ that is presumed to arise from different populations of electron spin orientations. This beating has been interpreted in a number of heterostructures $^{3-16}$ to deduce the Rashba parameter $\alpha$, which is expected to depend on materials parameters and the electric field. The zero-field spin splitting has also been inferred from Raman-scattering spectra ${ }^{17}$ in GaAs and weak anti-localization measurements in InGaAs quantum wells ${ }^{18}$.

In this paper, we report on electron spin resonance (ESR) experiments on 2DESs in InSb quantum wells. By observing transitions between states with the same Landau level index but opposite spin orientations, we directly measure the spin splitting as a function of applied magnetic field $B$. In addition to the $B=0$ spin splitting, the Rashba model ${ }^{1}$ predicts considerable modification of the Landau-level energy structure at nonzero B for asymmetric heterostructures, which has not been previously confirmed. Because of the large effective $g$ factor and large predicted Rashba effect, ${ }^{6}$ the spin splitting in InSb-based 2DESs should occur at far infrared frequencies for $\mathrm{B}<10 \mathrm{~T}$. Our experiments demonstrate that the Rashba effect is relatively strong in InSb quantum wells and that ESR is a powerful technique for studying the Rashba effect.

Our samples are InSb single quantum wells of widths 30 and $20 \mathrm{~nm}$ with $\mathrm{Al}_{0.09} \mathrm{In}_{0.91} \mathrm{Sb}$ barrier layers that are $\delta$ doped with $\mathrm{Si}$. The $\mathrm{Si} \delta$ layers are located either singly on one side of the quantum well (asymmetric samples) or equidistant on both sides of the quantum well (symmetric samples). The $\delta$-doped layers within the barrier layers are typically located $70 \mathrm{~nm}$ from the well center. The barrier layers on the substrate and surface sides of the wells are $3 \mu \mathrm{m}$ and $160 \mathrm{~nm}$ thick, respectively. Silicon dopants are placed near the surface to limit surface depletion. Thus, we expect the shape and symmetry of the wells to be determined only by the well/barrier mismatch (symmetric in all samples) and the mismatch in doping layers. In this work, seven symmetric and four asymmetric samples were studied. The electron concentrations in the wells $\left(n_{s}\right)$ are in the range $(0.95-3.6) \times 10^{11} \mathrm{~cm}^{-2}$, where only the ground-state subband is occupied. The mobilities of the samples studied range from $70000 \mathrm{~cm}^{2} / \mathrm{Vs}$ to $150000 \mathrm{~cm}^{2} / \mathrm{Vs}$. The energygap discontinuity ${ }^{19}$ as well as the band offsets ${ }^{20}$ are well characterized in this strained-layer system.

It has been shown theoretically that a lack of inversion symmetry and a small energy gap can make electric-dipole

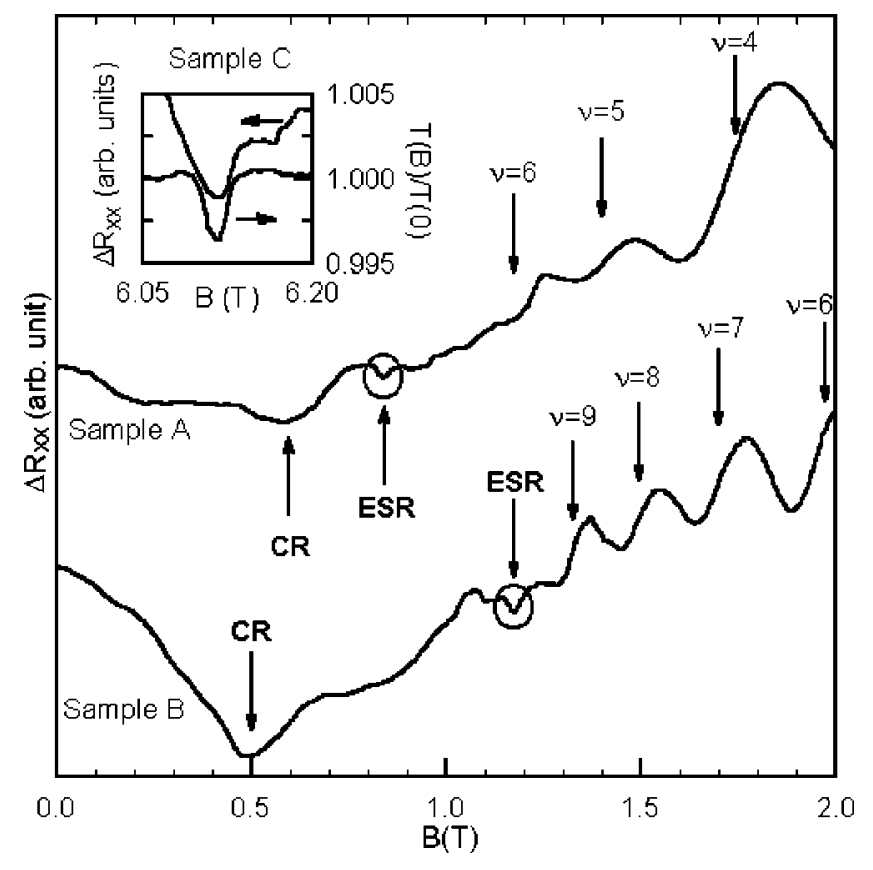

FIG. 1. Change in resistance due to FIR illumination as a function of applied magnetic field for Sample A (asymmetric) and Sample B (symmetric). Features attributed to cyclotron resonance (CR) and electron spin resonance (ESR) are labeled. The number of filled spin-split Landau levels $\nu$ is indicated for some B values where $\nu$ is an integer. Inset: ESR in Sample C as measured in the amount of FIR radiation transmitted $\mathrm{T}(\mathrm{B}) / \mathrm{T}(0)$ and in $\Delta R_{x x}$. 
TABLE I. The list of the samples studied in this work to probe the electron spin resonance in a wide range of magnetic fields and wavelengths. The symbols a and s are for asymmetric and symmetric confinement potentials, respectively.

\begin{tabular}{|c|c|c|c|c|c|c|}
\hline Sample & Symmetry & $\begin{array}{c}\mathrm{W} \\
(\mathrm{nm})\end{array}$ & $\begin{array}{c}\lambda \\
(\mu \mathrm{m})\end{array}$ & $\begin{array}{c}\text { Tilt } \\
\text { (degrees) }\end{array}$ & $\begin{array}{c}n_{s}\left(\times 10^{11}\right) \\
\left(\mathrm{cm}^{-2}\right)\end{array}$ & $\begin{array}{l}\mathrm{B} \\
(\mathrm{T})\end{array}$ \\
\hline S285 & $\mathrm{a}$ & 30 & 184 & 50 & 1.9 & 3.5 \\
\hline S285 & $\mathrm{a}$ & 30 & 184 & 30 & 1.9 & 3.1 \\
\hline S285 & $\mathrm{a}$ & 30 & 432 & 30 & 1.9 & 1.15 \\
\hline S360 & $\mathrm{a}$ & 30 & 119 & 45 & 2.5 & 5.9 \\
\hline S360 & $\mathrm{a}$ & 30 & 458 & 30 & 2.7 & 1.05 \\
\hline S360 & $\mathrm{a}$ & 30 & 458 & 45 & 2.2 & 0.9 \\
\hline S356 & $\mathrm{a}$ & 20 & 96.5 & 30 & 1.5 & 5.56 \\
\hline S356 & $\mathrm{a}$ & 20 & 163 & 45 & 1.6 & 3.5 \\
\hline S356 & $\mathrm{a}$ & 20 & 184 & 50 & 1.2 & 3.1 \\
\hline S356 & $\mathrm{a}$ & 20 & 418 & 45 & 1.1 & 1.0 \\
\hline S356 & $\mathrm{a}$ & 20 & 432 & 45 & 1.3 & 0.85 \\
\hline S707 & a & 20 & 184 & 30 & 2.3 & 3.2 \\
\hline S707 & $\mathrm{a}$ & 20 & 432 & 30 & 2.3 & 0.6 \\
\hline S644 & $\mathrm{s}$ & 30 & 184 & 30 & 2.2 & 3.35 \\
\hline S377 & $\mathrm{s}$ & 30 & 119 & 45 & 2.6 & 6.1 \\
\hline S377 & $\mathrm{s}$ & 30 & 418 & 50 & 2.8 & 1.9 \\
\hline S499 & $\mathrm{s}$ & 30 & 458 & 45 & 2.1 & 1.35 \\
\hline S716 & $\mathrm{s}$ & 30 & 96.5 & 30 & 1.4 & 5.67 \\
\hline S716 & $\mathrm{s}$ & 30 & 163 & 50 & 1.4 & 3.95 \\
\hline S710 & $\mathrm{s}$ & 30 & 119 & 30 & 0.9 & 4.35 \\
\hline S372 & $\mathrm{s}$ & 30 & 184 & 50 & 3.7 & 4.05 \\
\hline S704 & $\mathrm{s}$ & 20 & 184 & 50 & 1.1 & 3.35 \\
\hline S704 & $\mathrm{s}$ & 20 & 119 & 30 & 1.2 & 4.1 \\
\hline S704 & $\mathrm{s}$ & 20 & 96.5 & 30 & 1.2 & 5.58 \\
\hline
\end{tabular}

spin resonance possible. ${ }^{21,22}$ In narrow gap semiconductors with large spin-orbit coupling, the strong interaction between the conduction and valence bands leads to mixing of the electronic energy states. The wave functions (with a nominal spin polarity and a Landau quantum number $n$ ) contain both spin orientations as well as Landau indices of $n$ and $n \pm 1 .{ }^{21,22}$ This situation allows for the occurrence of electric-dipole transitions between states of nominally different spin polarity. Electric-dipole-excited electron spin resonance was observed in $\mathrm{Hg}_{1-x} \mathrm{Cd}_{x}$ Te (Ref. 23) and later in bulk InSb by McCombe and Wagner in the far infrared (FIR) spectral range. ${ }^{24}$

We detect ESR at $4.2 \mathrm{~K}$ either in magneto-transmission of FIR radiation or by observing the laser-induced change in magnetotransport. ${ }^{25,26}$ In previous experiments on InSb inversion layers, ${ }^{27}$ a very small ESR signal was observed when the sample was normal with respect to the magnetic field axis, which also coincides with the direction of the incoming laser radiation. Their ESR signal was increased with tilt angle up to an order of magnitude at a $45^{\circ}$ tilt angle. In our experiment, the ESR signal was only above the detection limit when the tilt angle was between $30^{\circ}$ and $50^{\circ}$. We vary the tilt angle to extend the range of magnetic fields where the ESR is observable for a given electron density. In this case, the spins (and ESR) respond to the total B but the 2D Landau quantization (and thus the $\mathrm{SdH}$ oscillations and cyclotron resonance) depends only on the component of $\mathrm{B}$ along the sample normal.

Figure 1 shows the observed spin and cyclotron resonance minima for Sample A (a $20 \mathrm{~nm}$-wide asymmetric sample at a tilt angle of $\theta=45^{\circ}$ and with $\lambda=432 \mu \mathrm{m}$ incident radiation) and Sample B (a $30 \mathrm{~nm}$-wide symmetric sample with $\theta=45^{\circ}$ and $\left.\lambda=458 \mu \mathrm{m}\right) . \Delta R_{x x}$ is the difference between the resistance with and without incident FIR radiation. The positions of the other minima seen in this figure are independent of $\lambda$ and are therefore nonresonant. Bolometric effects are responsible for at least some of these nonresonant features. ${ }^{28}$ The data show that the ESR feature for Sample A is at a lower magnetic field than for Sample B. If only the Zeeman effect were important, we would expect the opposite to be true because the FIR frequency is higher and the well width is smaller for Sample A.

This discrepancy implies that there is an additional mechanism for spin splitting that is stronger in Sample A. The inset to Figure 1 compares the ESR signal observed in transmission with that observed in modulated magnetotransport for Sample C, a symmetric sample with $\theta=45^{\circ}$ and $\lambda=119 \mu \mathrm{m}$. The resonance is very narrow in both cases, on the order of $40 \mathrm{mT}$, similar to ESR in GaAs. ${ }^{25}$ By using samples with different electron densities, fixed wedges with 

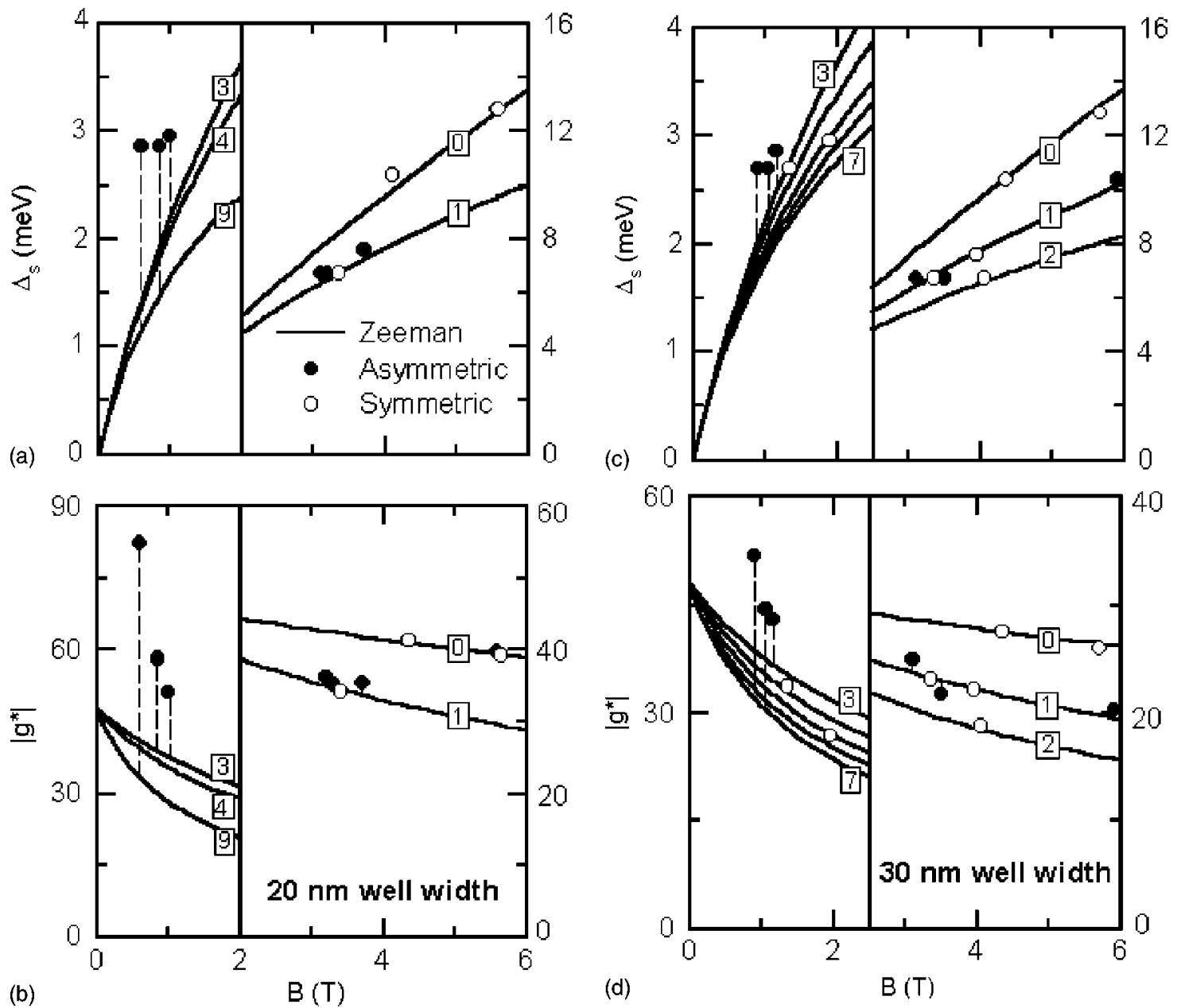

(b)

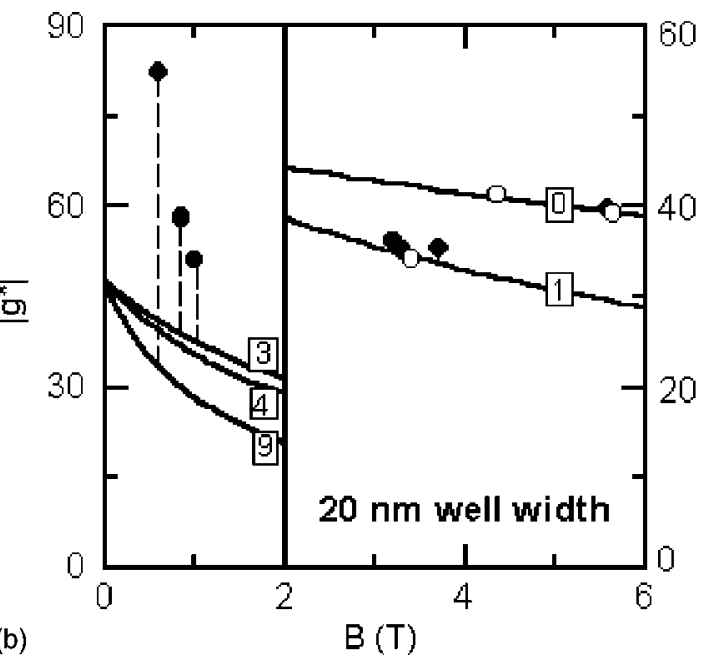

FIG. 2. Spin splitting and apparent $g$ factors for symmetric and asymmetric samples. Panels (a) and (b) are for $20 \mathrm{~nm}$ wells and (c) and (d) for $30 \mathrm{~nm}$ wells. The solid curves are a single-parameter fit to the data for symmetric samples, calculated using a modified Pidgeon and Brown model that does not include Rashba splitting. The curves are labeled with their Landau level indices. The solid and open circles represent the measured ESR data for the asymmetric and symmetric samples, respectively. Dashed lines connect data points to curves with the same Landau level index.

different tilt $\left(30^{\circ}, 45^{\circ}\right.$, and $\left.50^{\circ}\right)$, and seven laser lines $(\lambda=458,432,418,184,163,119$, and $96.5 \mu \mathrm{m})$ producing a sufficient signal-to-noise ratio, we are able to follow the ESR over a wide range of $\mathrm{B}$ and $n$. Table I summarizes the experimetal parameters of all the samples studied.

The observed spin splitting $\Delta s(\mathrm{~B})$ for the symmetric samples is plotted in Fig. 2(a), for wells that are $20 \mathrm{~nm}$ wide, and Fig. 2(c), for wells that are $30 \mathrm{~nm}$ wide. Also plotted in these figures is the $\Delta s(\mathrm{~B})$ expected in the absence of Rashba splitting from a modified bulk band structure ${ }^{29}$ that takes into account the extreme nonparabolicity of the InSb band structure. In this model, the bulk energy gap is replaced by the effective energy gap in the $2 \mathrm{D}$ band structure, i.e., by the energy spacing between the ground state valence- and conduction-band subbands. ${ }^{30}$ This effective energy gap depends on well width as does the momentum matrix element, which we use as the lone fitting parameter (within its accepted range of values ${ }^{31}$ ). All the other band parameters are fixed at their accepted values. The small Hartree potential results in a negligibly small effect of symmetry on the effective mass $\left(m^{*}\right)$ and the $g$ factor $(g *)$.
At $\mathrm{B}=0$ there is a reduction in the model InSb $g$ factor from -51 to -48 due in nearly equal parts to the change in energy gap from both the strain present in the InSb quantum well and from the subband quantization. The band edge effective mass is $0.014 m_{0}$ where $m_{0}$ is the free electron mass. Since we measured the electron density for each sample using $\mathrm{SdH}$ oscillations, we can deduce the highest occupied Landau level for each ESR data point. For the symmetric samples, $n$ for each point is the same as for the solid line that intersects it. We conclude therefore that our symmetric samples behave entirely as expected for wells with no Rashba splitting and that the resulting $g$ factors are the bare $g$ factors based on one-electron energy levels. Similar results have been reported for GaAs quantum wells. ${ }^{32,33}$

The asymmetric samples show quite different behavior, especially at low magnetic fields, as can be seen in Fig. 2. For the asymmetric samples, a dashed line connects each data point to the solid line that indicates the highest occupied Landau level. The spin splitting is observed to deviate from those values expected for a symmetric well to values even beyond those calculated using the band-edge $g$ factor, -48 . These deviations are more dramatic if we extract an apparent 


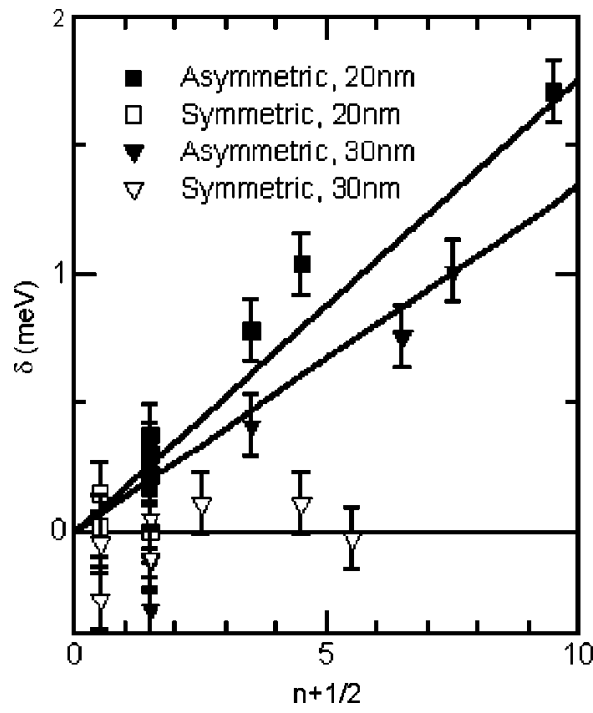

FIG. 3. Asymmetry induced spin splitting as a function of Landau level index. Values for $\delta$ are deduced by subtracting the results of the model calculation from the measured spin splitting. Two lines of best fit through the origin are drawn for the asymmetric samples with well widths of 20 and $30 \mathrm{~nm}$.

$g$ factor, $|g *| \equiv \Delta_{s}(\mathrm{~B}) / \mu_{B} \mathrm{~B}$. Figures 2(b) and 2(d) show that, as the magnetic field decreases, this apparent $g$ factor far exceeds the magnitude of the bulk value of the (negative) $g$ factor and the (lower) values predicted by the nonparabolic calculation. (If the measured spin splitting $\Delta s(\mathrm{~B})$ approaches a nonzero value at $\mathrm{B}=0$, as appears to be the case in Figs. 2(a) and 2(c), then the apparent $g$ factor diverges as B approaches zero.) Of course, our use of an apparent $g$ factor only serves to emphasize the departure from the spin splitting for the symmetric samples.

For the asymmetric samples the spin splitting is determined by the applied field (the Zeeman term) plus an effective field from the Rashba effect. The extrapolation of the measured spin splitting to zero magnetic field probes the predicted $^{1}$ splitting of the spin-up (+) and spin-down (-) subband energy dispersion

$$
E_{i \pm}(k)=E_{i} \pm \alpha k,
$$

where $E_{i}(k)$ is the subband dispersion in the absence of Rashba splitting, $\alpha$ is the Rashba spin-splitting parameter, and $k$ is the magnitude of the wavevector in the 2D layer. The zero-field spin splitting is then given by $\Delta s(0)=2 \alpha k_{F}$, where $\mathrm{k}_{F}$ is Fermi wave vector.

In addition to the zero-field spin splitting discussed above, Bychkov and Rashba ${ }^{1}$ also predicted a shift in the energy levels at $\mathrm{B}>0$ in asymmetric quantum wells. From this Landau-level dependent shift, the magnitude of the asymmetry induced component of the spin splitting is easily shown for $\mathrm{B} \gg \mathrm{B}_{\min }$ to $\mathrm{be}^{26,34}$

$$
\delta \cong 8 m * m_{0} \alpha^{2}(n+1 / 2) /\left[\hbar^{2}\left(2 m_{0}-m^{*} g *\right)\right]
$$

Here $m^{*}$ is the effective mass and $m_{0}$ the free electron mass. All our spin resonance observations occur at fields well in excess of $\mathrm{B}_{\text {min }}$ given by

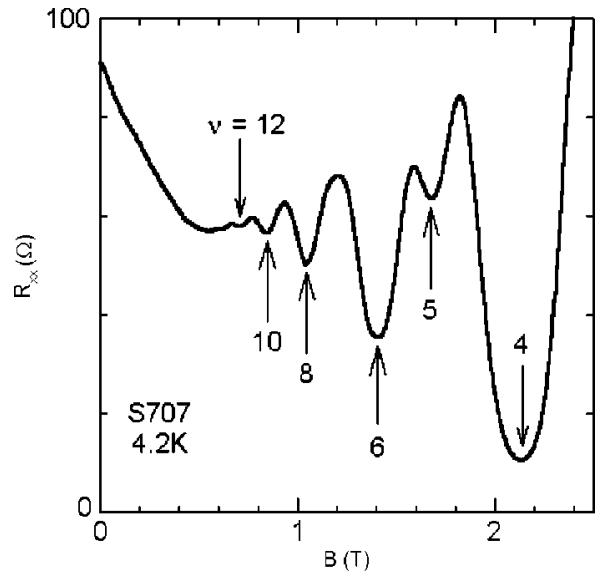

FIG. 4. Shubnikov de Haas oscillations in magnetoresistance versus applied magnetic field for an asymmetric sample. Several spin-split Landau level filling factors $(\nu)$ are indicated.

$$
B_{\min }=32 n\left(m * m_{0} \alpha\right)^{2} /\left[e \hbar^{3}\left(2 m_{0}-m * g *\right)^{2}\right],
$$

where $e$ is the electron charge. With $\alpha$ values found below and using the band-edge values of $m^{*}$ and $g^{*}$, we find $\mathrm{B}_{\min } \sim 0.01 \mathrm{~T}$. Thus we expect the B-independent expression (2) to be valid if we neglect nonparabolicity.

We determine experimental $\delta$ values from Figs. 2(a) and 2 (c) by taking the difference between the measured $\Delta s(\mathrm{~B})$ in a given sample and the $\Delta s(\mathrm{~B})$ value predicted for a symmetric sample at the same $\mathrm{B}$ and $n$. These differences are plotted for the asymmetric and the symmetric samples in Fig. 3 against $(n+1 / 2)$. The symmetric samples have $\delta$ values clustered about zero showing again how well they are fit by the Pidgeon-Brown model.

In contrast, $\delta$ for the asymmetric wells increases with increasing $n$. Since $\alpha$ for the asymmetric samples is expected to depend on several factors including electron density and well width, it is not surprising that all the data do not lie on a single line. We fit two lines through these data since the points for the $20 \mathrm{~nm}$ wells appear to be systematically higher than the points for $30 \mathrm{~nm}$ wells. We do not detect any systematic density dependence, but more data points are required to unambiguously determine whether such a dependence exists. The error bars account for several sources of error, including the ESR line width and a very weak dependence of $\Delta s(\mathrm{~B})$ on tilt angle. ${ }^{27}$

We calculate the effect of nonparabolicity by examining the multipler of $\alpha^{2}(n+1 / 2)$ in Eq. (2). Although the calculated values of $g^{*}$ and $m^{*}$ depend on the Fermi level, the multiplier is not so sensitive, for a given well width. For example, $g^{*}=-28.4, m^{*}=0.0190 m_{o}$, and the multiplier is $0.059 m_{0} / \hbar^{2}$ for the $30 \mathrm{~nm}$ well with the highest Fermi level. The $30 \mathrm{~nm}$ well with the lowest Fermi level has $g^{*}=-40.6$, $m^{*}=0.0176 m_{o}$, and the multiplier is $0.052 m_{0} / \hbar^{2}$. For the $20 \mathrm{~nm}$ wells, the multiplier ranges from $0.061 m_{0} / \hbar^{2}$ to $0.071 m_{0} / \hbar^{2}$ for our sample parameters. This suggests that nonparabolicity introduces only a small deviation from linearity with $(n+1 / 2)$ in the parabolic Eq. (2). ${ }^{35}$ Therefore, we use Eq. (2) to estimate values for $\alpha$ from the experimentally determined linear slopes shown in Fig. 3. With a multiplier 
value $0.066 m_{0} / \hbar^{2}$ for $20 \mathrm{~nm}$ wells $\left(0.056 m_{0} / \hbar^{2}\right.$ for $30 \mathrm{~nm}$ wells), we deduce an $\alpha$ value of $1.4 \times 10^{-9} \mathrm{eV} \mathrm{cm}$ for $20 \mathrm{~nm}$ wells $\left(1.3 \times 10^{-9} \mathrm{eV} \mathrm{cm}\right.$ for $30 \mathrm{~nm}$ wells $)$.

At first glance, it seems surprising that we can use the tilted B field to extract the Rashba splitting. Bychkov et al. predicted that the effect of spin-orbit interaction is reduced in a tilted magnetic field. ${ }^{36}$ The work of Pfeffer and Zawadzki, ${ }^{37}$ in contrast, predicts that the component of B in the plane of the sample is ineffective in quenching the Rashba splitting compared to the field component perpendicular to the sample plane. Furthermore, the Rashba splitting [Eq. (2)] depends on the field only indirectly through the observed $n$. From our experimental data, we cannot infer whether or not the Rashba effect depends strongly on tilt angle. In order to extract a quantitative measure of the Rashba parameter, we assume that the Rashba effect is unaffected by a parallel magnetic field, as the calculations of Ref. 37 predict.

Under the assumption of identical electrical forces causing the $2 \mathrm{D}$ spin-orbit coupling, the predicted ${ }^{38}$ ratios of $\alpha$ values for GaAs, InAs, and InSb quantum wells is 4.4:110:500. Recently, ${ }^{14} \alpha$ values ranging from $2 \times 10^{-9}$ to $4 \times 10^{-9} \mathrm{eV} \mathrm{cm}$ have been observed with gated InAs samples. This suggests that $\alpha$ values even larger than those reported here are achievable for InSb wells.

Many previous studies have cited beating in SdH oscillations as evidence for Rashba splitting. ${ }^{3-16}$ In our asymmetric (and symmetric) samples, no beating is observed in the $\mathrm{SdH}$ oscillations. ${ }^{26}$ The combination of a relatively low mobility and a large $g$ factor (spin splitting is resolved above $1.2 \mathrm{~T}$ ) restricts the range for observing beating to $0.5 \mathrm{~T}<\mathrm{B}<1.2 \mathrm{~T}$. The low electron densities result in only $\sim 5$ oscillations within this range. Thus, if beating were present it would be difficult to detect. Figure 4 shows the absence of beating in our most asymmetric structure. A strength of the ESR technique that we employ is its ability to detect spin-split levels even in systems with a low electron density and a relatively low mobility.

Our experimental observations were recently compared by Pfeffer and Zawadzki with the results of their three-level P. $\mathbf{p}$ model of $\mathrm{InSb} / \mathrm{Al}_{0.09} \mathrm{In}_{0.91} \mathrm{Sb}$ quantum-well structures. ${ }^{34}$ They calculated spin splitting due to both Zeeman and Rashba effects, assuming spin-dependent boundary conditions at the interfaces. The agreement between their calculation and our experimental data provides confirmation of the Bychkov-Rashba picture of spin-orbit coupling in our heterostructures.

In conclusion, we have demonstrated that ESR is a useful technique for studying spin splitting in InSb quantum wells. In asymmetric structures at low magnetic fields, we observed spin splitting that was significantly larger than predicted for the Zeeman effect. The additional splitting can be attributed to a strong Rashba effect.

The authors are grateful to Sheena Murphy for helpful comments, to Niti Goel for help with samples, and to Robert Meyer for experimental help. This work was supported by the National Science Foundation under Grant Nos. DMR9973167 and DMR-0080054.
*Author to whom correspondence should be addressed: Department of Physics, Virginia Tech, Blacksburg, VA 24061; electronic mail: khoda@vt.edu

${ }^{1}$ Y. A. Bychkov and E. I. Rashba, J. Phys. C 17, 6039 (1984); Y. A. Bychkov and E. E. Rashba, Pis'ma Zh. Eksp. Teor. Fiz. 39, 66 (1984) [JETP Lett. 39, 78 (1984)]

${ }^{2}$ E. I. Rashba, Fiz. Tverd. Tela (Leningrad) 2, 1224 (1960) [Sov. Phys. Solid State 2, 1109 (1960)]; E. I. Rashba and V. I. Sheka, in Landau Level Spectroscopy, Modern Problems in Condensed Matter Sciences, Vol. 27.1, (North-Holland, Amsterdam, 1991) pp. 132-206.

${ }^{3}$ H. L. Stormer, Z. Schlesinger, A. Chang, D. C. Tsui, A. C. Gossard, and W. Wiegmann, Phys. Rev. Lett. 51, 126 (1983).

${ }^{4}$ J. Luo, H. Munekata, F. F. Fang, and P. J. Stiles, Phys. Rev. B 38, 10142 (1988); J. Luo, H. Munekata, F. F. Fang, and P. J. Stiles, ibid. 41, 7685 (1990).

${ }^{5}$ B. Das, D. C. Miller, S. Datta, R. Reifenberger, W. P. Hong, P. K. Bhattacharya, J. Singh, and M. Jaffe, Phys. Rev. B 39, 1411 (1989).

${ }^{6}$ B. Das, S. Datta, and R. Reifenberger, Phys. Rev. B 41, 8278 (1990).

${ }^{7}$ G. Engels, J. Lange, Th. Schäpers, and H. Lüth, Phys. Rev. B 55, R1958 (1997).

${ }^{8}$ J. Nitta, T. Akazaki, H. Takayangi, and T. Enoki, Phys. Rev. Lett. 78, 1335 (1997).
${ }^{9}$ Th. Schäpers, G. Engels, J. Lange, Th. Klocke, M. Hollfelder, and H. Lüth, J. Appl. Phys. 83, 4324 (1998).

${ }^{10}$ J. P. Heida, B. J. van Wees, J. J. Kuipers, T. M. Klapwijk, and G. Gorghs, Phys. Rev. B 57, 11911 (1998).

${ }^{11}$ J. P. Lu, J. B. Yau, S. P. Shukla, M. Shayegan, L. Wissinger, U. Rössler, and R. Winkler, Phys. Rev. Lett. 81, 1282 (1998).

${ }^{12}$ C. Hu, J. Nitta, T. Akazaki, H. Takayangi, J. Osaka, P. Pfeffer, and W. Zawadzki, Phys. Rev. B 60, 7736 (1999).

${ }^{13}$ T. Matsuyama, R. Kürsten, C. Meibner, and U. Merkt, Phys. Rev. B 61, 15588 (2000).

${ }^{14}$ D. Grundler, Phys. Rev. Lett. 84, 6074 (2000).

${ }^{15}$ Y. S. Gui, C. M. Hu, Z. H. Chen, G. Z. Zheng, S. L. Guo, J. H. Chu, J. X. Chen, and A. Z. Li, Phys. Rev. B 61, 7237 (2000).

${ }^{16}$ X. C. Zhang, A. Pfeuffer-Jeschke, K. Ortner, V. Hock, H. Buhmann, C. R. Becker, and G. Landwher, Phys. Rev. B 63, 245305 (2001).

${ }^{17}$ B. Jusserand, D. Richards, G. Allan, C. Priester, and B. Etienne, Phys. Rev. B 51, 4707 (1995).

${ }^{18}$ T. Koga, J. Nitta, T. Akazaki, and H. Takayanagi, Phys. Rev. Lett. 89, 046801 (2002).

${ }^{19}$ N. Dai, F. Brown, R. E. Doezema, S. J. Chung, K. J. Goldammer, and M. B. Santos, Appl. Phys. Lett. 73, 3132 (1998).

${ }^{20}$ N. Dai, G. A. Khodaparast, F. Brown, R. E. Doezema, S. J. Chung, and M. B. Santos, Appl. Phys. Lett. 76, 3905 (2000).

${ }^{21}$ W. Zawadzki, Landau Level Spectroscopy, Modern Problems in 
Condensed Matter Sciences, Vol. 27.1, (North-Holland, Amsterdam, 1991) pp. 485-479.

${ }^{22}$ B. D. McCombe and R. J. Wagner, Intraband Magneto-Optical Studies of Semiconductors in the Far-Infrard, Advances in Electronics and Electron Physics, Vol. 37, (Academic, New York, 1975), pp. 1-78.

${ }^{23}$ B. D. McCombe, R. J. Wagner, and G. A. Prinz, Phys. Rev. Lett. 25, 87 (1970).

${ }^{24}$ B. D. McCombe and R. J. Wagner, Phys. Rev. B 4, 1285 (1971).

${ }^{25}$ D. Stein, K. v. Klitzing, and G. Weimann, Phys. Rev. Lett. 51, 130 (1983).

${ }^{26}$ G. A. Khodaparast, R. E. Doezema, S. J. Chung, K. J. Goldammer, and M. B. Santos, IPAP Conf. Series 2, 245 (2001)

${ }^{27}$ A. Därr, J. P. Kotthaus, and T. Ando, in Physics of Semiconductors, edited by F. G. Fumi (North-Holland, Amsterdam, 1976), pp. 774-777; A. Därr, Doctoral dissertation, Technische Universität München, 1978 (unpublished).

${ }^{28}$ N. G. Kalugin, G. Nachtwei, Yu. B. Vasilyev, S. D. Suchalkin, and K. Eberl, Appl. Phys. Lett. 81, 382 (2002).
${ }^{29}$ C. R. Pidgeon and R. N. Brown, Phys. Rev. 146, 575 (1966).

${ }^{30}$ M. J. Yang, R. J. Wagner, P. J. Lin-Chung, B. V. Shanabrook, J. R. Waterman, W. J. Moore, and J. L. Davis, Surf. Sci. 305, 271 (1994).

${ }^{31}$ Landolt-Börnstein, in Numerical Data and Functional Relationships in Science and Technology, edited by O. Madelung, Group III, Vol. 22, (Springer-Verlag Berlin, 1987).

${ }^{32}$ M. Dobers, K. v. Klitzing, and G. Weimann, Phys. Rev. B 38, 5453 (1988).

${ }^{33}$ R. Meisels, I. Kulac, F. Kuchar, and M. Kriechbaum, Phys. Rev. B 61, 5637 (2000).

${ }^{34}$ P. Pfeffer and W. Zawadzki, Phys. Rev. B 68, 035315 (2003).

${ }^{35}$ W. Zawadzki, Phys. Lett. 4, 190, (1963)

${ }^{36}$ Yu. Bychkov, V. I. Mel'nikov, and E. I. Rashba, Springer Series in Solid State Sciences, Vol. 101, High Magnetic Fields in Semiconductors III (Springer-Verlag, Berlin, 1992).

${ }^{37}$ P. Pfeffer and W. Zawadzki, Phys. Rev. B 59, R5312 (1999).

${ }^{38}$ E. A. de Andrada e Silve, G. C. La Rocca, and F. Bassani, Phys. Rev. B 55, 16293 (1997) 